\title{
IMPLANTAÇÃO DE UM SISTEMA CLASSIFICATÓRIO DO GRAU DE DEPENDÊNCIA DOS CUIDADOS DE ENFERMAGEM EM UM SERVIÇO DE EMERGÊNCIA
}

[ Implementation of a system classification in level of dependence nursing cares in emergency unit] [ Implantación de un sistema de clasificación del nivel de la dependencia de los cuidados de oficio de enfermería en una unidad de emergencia]

Juliana Helena M ontezeli*, A nabely de A breu L opes**

RESUMO: A procura pelos serviços de emergência cresceu acentuadamente nas últimas décadas. Contudo, o dimensionamento de pessoal de enfermagem para este setor continua sendo um grande desafio para as instituições de saúde. Este estudo foi desenvolvido no Pronto Socorro de um hospital-escola objetivando traçar o perfil da clientela segundo o grau de dependência dos cuidados de enfermagem, utilizando instrumento próprio. Foram classificados de junho a dezembro de 2002 os clientes atendidos na sala de emergência e os internados no setor ao aguardo de vagas em outras unidades. Constatou-se predominância de clientes grau III e IV internados e de grau I e II sendo atendidos na sala de emergência, comprovando a descaracterização do serviço e o desvio das funções emergenciais da equipe de enfermagem. 0 perfil da clientela encontrado proporcionou uma profunda reflexão da filosofia do Pronto Socorro e forneceu subsídios para o redimensionamento de pessoal de enfermagem pela assistência progressiva.

PALAVRAS-CHAVE: Enfermagem; Emergência; R ecursos humanos.

ABSTRACT: The demand for emergency services has increased sharply in the last decades. However nursing staff training for this specialization is still a great challenge for healthcare institutions. This study was performed in the emergency room of a teaching hospital, using own instruments; the objective was to develop a profile of the patients, according to the degree of dependence on nursing care. From J une to December 2002, both the patients treated in the emergency room and those admitted to this area while waiting for admittance in other units were classified. It was found a predominance of grade III and IV patients who were admitted at the emergency room and grade I and II patients who were treated there; at the same time, a lack of clear guidelines for action and a deviation from the nursing team's functions were evident. The patients' profile that was found provides an in-depth reflection on the philosophy of emergency care and establishes the bases for the training of nursing staff towards progressive care.

KEYWORDS: Nursing; Emergency; Human resources.

RESUMEN: La demanda de los servicios de urgencia se acentuó en las últimas décadas. La formación de los profesionales de enfermería para esa especialidad sigue siendo el gran desafío para las instituciones de salud. Utilizando instrumentos propios, el estudio se desarrolló en la sala de urgencias de un hospital. El objetivo era trazar un perfil de los pacientes según el grado de dependencia de los cuidados de enfermería. De junio a diciembre de 2002, fueron clasificados tanto los pacientes atendidos en la sala de urgencias, como los internados en este sector a la espera de plazas en otras unidades. Se constata la predominancia de pacientes de grado III y IV internados y de grado I y II atendidos en la sala de urgencias. A I mismo tiempo, se demuestra la fal ta de una pauta clara de actuación y un desvío en las funciones del equipo de enfermería. El perfil de los pacientes encontrado proporciona una profunda reflexión acerca de la filosofía de urgencias y proporciona las bases para la formación de los profesional es de enfermería hacia una asistencia progresiva. PALABRAS CLAVE: Enfermería; Emergência; Recursos Humanos.

*Enfermeira Coordenadora do Pronto Socorro do Hospital U niversitário Evangélico de Curitiba. Especialista em Projetos A ssistenciais de Enfermagem pela Universidade Federal do Paraná - UFPR. Especialista em Enfermagem de Emergência pela Pontifícia Universidade Católica do ParanáPUCPR.

** Enfermeira do Pronto Socorro do Hospital Universitário Evangélico de Curitiba. Especialista em Enfermagem de Emergência pela PUCPR. 


\section{INTRODUÇÃO}

A quantificação de recursos humanos de enfermagem constitui a etapa inicial para atender direta ou indiretamente às necessidades de assistência de determinada clientela, tendo por finalidade previsão da quantidade de funcionários por categoria requerida para 0 atendimento ${ }^{(1)}$. Entretanto, 0 dimensionamento de recursos humanos, em qual quer organização, tem sido considerado um desafio, pois é o mais complexo da organização, sendo que os demais recursos exigem a sua presença para que possam ser utilizados (2).

Desde décadas atrás, afirma-se que 0 grau de desenvolvimento sócio-econômico de um país está estreitamente relacionado ao seu contingente de pessoal para as atividades de saúde ${ }^{(3)}$. Em virtude das implicações que o dimensionamento inadequado de profissionais de enfermagem causa sobre 0 resultado da qualidade da assistência prestada, este assunto tem requerido a atenção dos enfermeiros, pois a irregularidade quali-quantitativa desses profissionais lesa a clientela dos serviços de saúde no seu direito de assistência livre de riscos, além de poder comprometer legalmente a instituição devido a falhas ocorridas no atendimento ${ }^{(2)}$.

A procura pelos serviços de emergência vem crescendo acentuadamente desde a década de 50 do século passado, tendendo a continuar aumentando. A imprevisibilidade da demanda aliada à gravidade e à complexidade torna esse cenário um verdadeiro desafio e um dos setores mais importantes de um hospital, onde a assistência prestada deve possuir uma qualidade de nível el evado e ser qualificada para todas as adversidades possíveis ${ }^{(4)}$.

Este estudo foi realizado em um Pronto Socorro de uma instituição hospitalar de ensino de Curitiba no Paraná que presta atendimento em emergências clínicas e cirúrgicas pelos sistemas público ou privado, sendo referência no atendimento de trauma na cidade. Com uma demanda média de 300 atendimentos/dia, é comum a permanência de clientes em observação ou internados no setor de emergência à espera de vagas nas unidades de internamento da instituição, a qual conta com um total de 585 leitos. N essas condições, permanecem clientes que necessitam de diferentes níveis de complexidade de atendimento pela enfermagem, incluindo cuidados intensivos.

$\mathrm{Na}$ vivência cotidiana como enfermeiras responsáveis pel o serviço de emergência deste hospitalescola, observamos uma mudança no nível de complexidade nos clientes atendidos, com aumento progressivo na gravidade dos mesmos. Entretanto, não tínhamos dados científicos que comprovassem essa percepção, dificultando o redimensionamento de pessoal para a equipe de enfermagem.
A competência do dimensionamento de pessoal de enfermagem é do enfermeiro que atua diretamente na assistência, pois este identifica e reavalia continuamente os recursos existentes e as necessidades da clientela. Entretanto, na prática, a falta de parâmetros bem definidos destinados a este dimensionamento acaba por fragilizar as argumentações da chefia frente à cúpula administrativa da instituição ${ }^{(5)}$. A ssim, o grau de dependência representa um parâmetro fundamental para viabilizar um dimensionamento de recursos humanos de enfermagem condizente com a complexidade de cuidados inspirada pela clientela.

Neste contexto de dificuldades assistenciais e gerenciais decorrentes da falta de instrumentos específicos que conduzissem e justificassem as propostas de redimensionamento de pessoal em aspectos quantitativos e qualitativos, emergiu a necessidade de implantação de um método classificatório dos clientes atendidos no pronto socorro em questão, com o intuito de traçar o perfil dessa clientela e futuramente adequar o quadro de funcionários de enfermagem a um número mais próximo do ideal calculado pelo método da assistência progressiva ao cliente.

\subsection{OBJETIV 0}

Identificar o grau de dependência do cuidado de enfermagem dos clientes atendidos de junho a dezembro de 2002 no Pronto Socorro de um hospital-escola de Curitiba-PR, delineando o nível de complexidade da clientela.

\section{REVISÃO DE LITERATURA}

As instituições hospitalares têm procurado desenvolver estratégias que conciliem as dificuldades diversas e a manutenção da qualidade assistencial (6). A s questões de quantificação de pessoal na enfermagem sempre estiveram presentes no discurso das instituições hospitalares, entretanto, não são traduzi das em decisões concretas para a implantação de um dimensionamento ideal (7). Existem ainda outras vertentes que podem complementar e fundamentar cientificamente a classificação de pacientes como política institucional que se reflete no processo de trabalho da enfermagem, como a carga horária de trabalho, o quantitativo insuficiente de recursos humanos e também a verticalização do organograma institucional, cabendo ao enfermeiro buscar mudanças para reversão deste quadro, melhorando 0 atendimento prestado ${ }^{(8)}$.

A preocupação com a excelência da assistência é revelada pela história, pois desde os tempos Nightingaleanos, Florence buscava colocar os pacientes que necessitavam de maiores cuidados mais próximos 
ao posto de enfermagem ${ }^{(9)}$. A tualmente, considera-se que o sistema de classificação de pacientes é um método para viabilizar o cuidado individualizado com o intuito de melhorar os padrões de qualidade assistencial ${ }^{(6)}$.

A determinação do nível de complexidade para os cuidados de enfermagem depende da anál ise de todas as variáveis implícitas na totalidade do ser humano ${ }^{(10)}$. Este processo demonstra ser fundamentado e solidificado, pois que necessita ser edificado em evidências analisadas individualmente, visando determinar, validar e monitorar o cuidado individualizado ${ }^{(7)}$. Dessa forma, a quantificação de pessoal de enfermagem depende não somente do conhecimento da carga de trabalho existente nas unidades como também das necessidades de assistência dos clientes e do padrão de cuidado pretendido (11).

A primeira geração de sistema de classificação de pacientes teve origem nos Estados U nidos, em 1960, com o objetivo de dimensionar os recursos humanos em enfermagem e, somente em sua terceira geração, apresentou estrutura que permitia indicar as necessidades de cuidados, tempo real da assistência, complexidade assistencial e formação necessária para proporcionar o cuidado, além de favorecer 0 conhecimento do perfil dos pacientes de determinada unidade e impulsionar a qualidade assistencial de enfermagem ${ }^{(8)}$.

No Brasil, o primeiro modelo de dimensionamento de pessoal de enfermagem a considerar a variação de gravidade dos pacientes foi 0 de Riberio ${ }^{(12)}$. O utros autores ${ }^{(13,14)}$ utilizaram este critério de classificação de pacientes segundo as necessidades de cuidados para estabelecer o número de horas de enfermagem.

A tualmente, um estudo para validar um instrumento de classificação do grau de dependência de cuidados de quatro níveis em um hospital-escola da Região N orte do Paraná concluiu, entre outros aspectos, que a validação do instrumento proporcionou aos enfermeiros uma análise profunda no processo de observação das necessidades dos pacientes, tornando-se uma ferramenta de trabal ho eficiente na prática diária ${ }^{(15)}$. A lém disso, salienta-se que a classificação do paciente vem a fornecer ao enfermeiro um panorama da si tuação da assistência, sendo importante para o planejamento do cuidado e al ocação de recursos humanos e materiais, sendo esboço da demanda assistencial (8).

\section{METODOLOGIA}

Trata-se de um estudo quantitativo, desenvolvido na unidade de Pronto Socorro de uma instituição hospitalar de ensino da cidade de Curitiba no Paraná, durante os meses de junho a dezembro de 2002, no qual os clientes atendidos na sala de emergência e os que permaneceram em observação/internados foram classificados segundo o grau de dependência de cuidados de enfermagem, sendo aprovado pelo Comitê de Ética, Diretoria e Gerência de Enfermagem da instituição.

Neste estudo foi realizada a primeira etapa do dimensionamento de pessoal de enfermagem, fundamentando-se no trabalho de Kurcgant, Cunha e Gaidzinski, que compreende a caracterização da clientela ${ }^{(1)}$.

A indicação do critério de assistência progressiva pode ser aplicada a qualquer instituição de saúde, podendo 0 enfermeiro adaptar o sistema de classificação às possibilidades institucionais (2). Para classificar os pacientes do pronto socorro em questão foi realizada uma adaptação do sistema classificatório proposto e validado por Martins e Haddad ${ }^{(15)}$, de modo a suprir as necessidades dos clientes que aportam ao serviço de emergência.

Os clientes da unidade foram divididos em duas categorias distintas: a primeira categoria contemplada pelos pacientes atendidos na sala de emergência e a segunda pelos pacientes em observação ou internados no aguardo de vagas em outros setores. Para cada uma das categorias foram aplicados instrumentos classificatórios semelhantes, porém com direcionamento e características próprias para cada situação, como pode ser conferido nos Quadros 1 e 2.

Todos os atendimentos no período do estudo realizados na sala de emergência foram classificados, sendo somados no final do período de vinte e quatro horas.

U ma diferença importante de ser mencionada entre a classificação dos clientes emergenciais e dos internados ou em observação é que nesta última a classificação ocorreu apenas uma vez durante 0 dia, demonstrando uma amostra de toda a demanda, sendo realizada pelo enfermeiro do turno da manhã, imediatamente após o recebimento do plantão.

Todo o processo classificatório foi baseado em entrevista e exame físico dos clientes, além dos casos em que já havia diagnóstico médico estabelecido. No final de cada mês, os números de cada categoria foram somados em cada um dos níveis de complexidade, tornando possível delinear o perfil da clientela segundo o grau de dependência de cuidados de enfermagem.

\section{RESULTADOS}

A classificação dos atendimentos real izados na sala de emergência seguiu os critérios rel acionados no Quadro 1, descritos a seguir, enquanto os clientes em observação ou internados no setor aguardando vagas em outras unidades foram classificados segundo os critérios apontados no Quadro 2, ambos contendo quatro níveis de complexidade. 
Quadro 1 - Escala classificatória do grau de dependência de cuidados de enfermagem dos clientes atendidos na sala de emergência do Pronto Socorro. Curitiba, 2002

\begin{tabular}{|c|l|l|}
\hline Grau de dependência & \multicolumn{1}{|c|}{ I } & \multicolumn{1}{|c|}{ II } \\
\hline Características do cliente & $\begin{array}{l}\text { Sem desvios de comportamento } \\
\text { Sutura simples } \\
\text { Imobilização cervical profilático } \\
\text { Contusões leves }\end{array}$ & $\begin{array}{l}\text { Com ou sem desvios de comportamento } \\
\text { Observação moderada do estado geral } \\
\text { Escala de Glasgow 15 a 13 } \\
\text { Escala de trauma 11 e 12 } \\
\text { Imobilização cervical } \\
\text { Contusões leves } \\
\text { Suturas simples } \\
\text { Oxigenoterapia }\end{array}$ \\
\hline Grau de dependência & \multicolumn{1}{|c|}{ III } & IV \\
\hline Características do cliente & $\begin{array}{l}\text { Com desvio de comportamento } \\
\text { Observações freqüentes do estado geral } \\
\text { Escala de Glasgow 12 a 9 } \\
\text { Escala de trauma 7 a 10 } \\
\text { Imobilização cervical e/ou outras } \\
\text { Terapia endovenosa } \\
\text { Fraturas fechadas } \\
\text { Suturas complexas } \\
\text { Monitoração freqüente de dados vitais }\end{array}$ & $\begin{array}{l}\text { Inconscientes ou com desvios no padrão de comportamento } \\
\text { Escala de Glasgow 8 a 3 } \\
\text { Imobilização cervical e/ou outras } \\
\text { Fraturas expostas } \\
\text { Terapia endovenosa contínua } \\
\text { Transfusão de hemoderivados de emergência } \\
\text { Ferimentos por arma de fogo } \\
\text { Ferimentos por arma branca } \\
\text { Insuficiência respiratória } \\
\text { Requer medidas complexas para a manutenção da vida } \\
\text { Monitoração freqüente dos dados vitais } \\
\text { Total dependência da enfermagem }\end{array}$ \\
\hline
\end{tabular}

Fonte: dados da pesquisa

Quadro 2 - Escala classificatória do grau de dependência de cuidados de enfermagem dos clientes em observação/ internados no Pronto Socorro. Curitiba, 2002

\begin{tabular}{|c|l|l|}
\hline Grau de dependência & \multicolumn{1}{c|}{ I } & \multicolumn{1}{c|}{ II } \\
\hline Características do cliente & $\begin{array}{l}\text { Sem desvios de comportamento } \\
\text { Deambula, higieniza-se e alimenta-se sozinho } \\
\text { Medicação VO, tópica, IM } \\
\text { Controle de SSVV 3X dia } \\
\text { Sutura simples }\end{array}$ & $\begin{array}{l}\text { Com ou sem desvios de comportamento } \\
\text { Deambula, higieniza-se e alimenta-se sozinho } \\
\text { Medicação IM e/ou EV } \\
\text { Cuidados pré-operatórios simples } \\
\text { Preparo simples para exames } \\
\text { Cuidados pós-procedimentos invasivos } \\
\text { Controle de SSVV 3X dia }\end{array}$ \\
\hline Grau de dependência & III & \multicolumn{1}{c|}{ IV } \\
\hline Características do cliente & $\begin{array}{l}\text { Auxilio para deambular, higiene e alimentação } \\
\text { Medicação IM e/ou EV } \\
\text { Cuidados pré e pós-operatórios complexos } \\
\text { Controle de SSVV 4/4h } \\
\text { Controle de diurese } \\
\text { Observações freqüientes das condições do } \\
\text { comportamento } \\
\text { Alimentação via sonda }\end{array}$ & $\begin{array}{l}\text { Inconscientes ou com desvios no padrão de } \\
\text { comportamento } \\
\text { Acamado continuamente, higiene no leito } \\
\text { Terapia endovenosa contínua } \\
\text { Requer medidas complexas para manutenção da } \\
\text { vida } \\
\text { Total dependência da enfermagem }\end{array}$ \\
\hline
\end{tabular}

Fonte: dados da pesquisa

Cogitare Enferm 2006 set/dez; 11(3):239-44 
A Tabela 1 descreve o resultado da classificação de pacientes segundo o grau de dependência de cuidados durante os meses do estudo, tanto no que diz respeito aos atendimentos em sala de emergência quanto aos casos de internamento ou em observação dos clientes. Visualiza-se uma predominância de graus III e IV entre os internados/ em observação e graus I ell entre os atendimentos em sala de emergência.

Tabela 1 - Graus de dependência de cuidados dos clientes atendidos na sala de emergência internados ou em observação no Pronto Socorro de junho a dezembro de 2002. Curitiba

\begin{tabular}{ccc}
\hline Grau & $\begin{array}{c}\text { Sala de } \\
\text { Emergência }\end{array}$ & Internados/Obervação \\
\hline I & 915 & 751 \\
II & 1097 & 1388 \\
III & 678 & 1222 \\
IV & 804 & 1512 \\
\hline Total & 3494 & 4873 \\
\hline
\end{tabular}

A o acompanharmos a Tabela 1, podemos visualizar que há um fluxo inadequado dos clientes atendidos no Pronto Socorro em questão, ou seja, uma vez atendidos, os clientes graves tendem a permanecer no serviço de emergência, impossibilitando outros atendimentos graves. Possivelmente, essa situação advém da demanda extremamente numerosa que aponta ao serviço ser incompatível com o número de leitos de internamento, principalmente aos pacientes com necessidade de cuidados intensivos.

Os percentuais demonstrados na Gráfico 1 confirmam que neste Pronto Socorro há mais clientes graves internados ou em observação $(25 \%$ grau III e $32 \%$ grau IV) do que sendo atendidos na sala de emergência ( $26 \%$ grau I e $32 \%$ Grau II).

D etecta-se, assim, uma descaracterização do serviço de emergência, uma vez que a presença de clientes internados no setor implica na necessidade da equipe de enfermagem dispensar cuidados característicos de unidades de internamento e/ou de terapia intensiva.

$N$ esse sentido, as ações assistenciais da equipe de enfermagem tendem a se desviar dos cuidados emergenciais para assistir aos clientes que permanecem no setor, seja por problemas clínicos, cirúrgicos ou por traumatismos.

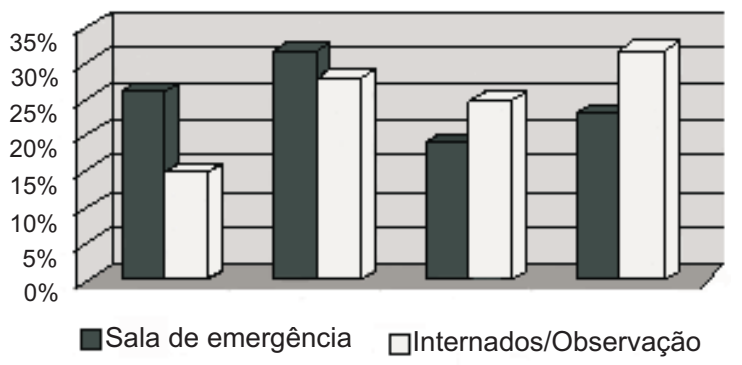

Gráfico 1 - G raus de dependência de cuidados dos clientes atendidos na sala de emergência e internados/ observação no Pronto Socorro de junho a dezembro de 2002. Curitiba

Quando analisamos os números totais dos clientes classificados a partir da soma dos internados/em observação com os atendimentos na sala de emergência, verificamos que há um equilíbrio entre as quatro categorias classificatórias.

Tabela 2 - Graus de dependência totais dos clientes do Pronto Socorro de junho a dezembro de 2002. Curitiba

\begin{tabular}{cc}
\hline Grau & $\mathbf{N}^{0}$. de Clientes \\
\hline I & 1666 \\
II & 2485 \\
III & 1900 \\
IV & 2316 \\
\hline Total & 8367 \\
\hline
\end{tabular}
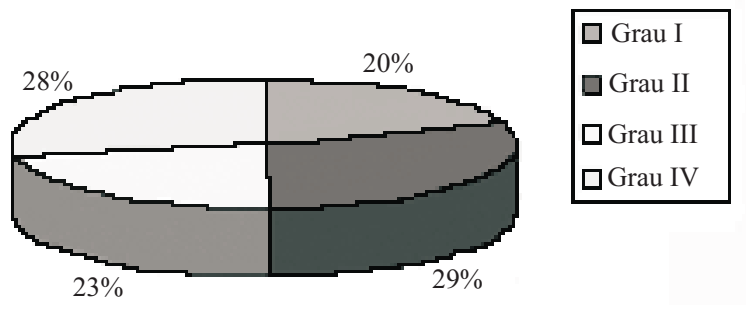

Gráfico 2 - Graus de dependência totais dos clientes do Pronto Socorro de junho a dezembro de 2002. Curitiba 
Salientamos, a partir dos dados apresentados na Tabela 2 e Gráfico 2, que $49 \%$ da clientela deste pronto socorro pertencem aos graus I ell. N uma análise secundária e dedutiva, afirmamos que esta grande porção de clientes graus I e II, considerados de complexidade leve, é devida à permanência neste setor de clientes graus III e IV ao aguardo de vagas nas unidades de internamento ou intensivas, culminando no desvio das funções emergenciais da equipe de enfermagem para a prática cuidativa dos internados.

\section{CONSIDERAÇÕESFINAIS}

Este estudo nos permitiu concluir que, no Pronto Socorro onde desenvolveu-se esta pesquisa, há uma clientela expressiva de perfil classificado entre os níveis III e IV, ou seja, de alta complexidade para as ações de enfermagem, em sal as de observação, enquanto os atendimentos realizados na sala deemergência classificam-se como graus I ell. Estequadro explica-se pelo fato de que, uma vez havendo grande número de clientes graves estagnados, não há condições de outros atendimentos complexos serem realizados.

Evidenciamos com as fontes bibliográficas estudadas que se trata de um assunto antigo que permeia a realidade da enfermagem até os dias de hoje. M uitos serviços encontram dificuldades eadversidades para traçar o perfil de sua clientela segundo o grau de dependência dos cuidados em enfermagem, sendo que a realização deste estudo e implantação do método fez-se possível no Pronto Socorro em questão graças à divisão do processo classificatório com os técnicos em enfermagem da unidade, os quais se dispuseram ao treinamento e colaboração durante todo o processo, demonstrando a importância da integração das várias categorias da enfermagem.

Dessa forma, sali ientamos a importância da val orização dos membros da equipe nas mudanças e inovações dentro das instituições, com 0 intuito de se alcançar a excelência na assistência de enfermagem, buscando um processo cuidativo individualizado, personalizado e progressivo ao cliente.

Convém lembrar que os instrumentos criados foram implantados na unidade, permitindo a continuidade do estudo, inclusive a comparação sazonal dos níveis de complexidade dos atendimentos, podendo dar segmento para o delineamento do perfil dos atendimentos de forma ainda mais profunda.

Notamos com os dados obtidos na pesquisa, uma descaracterização deste serviço de emergência e, conseqüentemente, um desvio das funções emergenciais da equipe de enfermagem para assistir os clientes internados ou em observação. Os dados desse estudo são preliminares eforam disponibilizados como subsídio à Gerência de Enfermagem para o cál culo de dimensionamento de recursos humanos pelo modelo de assistência progressiva. Paralelamente, ocasionou a gênese de uma reflexão mais profunda a respeito da filosofia e contextualização do serviço emergencial, permitindo sugerir à instituição a busca de uma forma mais viável de fluxo dos clientes graves após 0 atendimento de emergência, a fim de garantir a qualidade assistencial dispensada às categorias aqui estudadas.

Conclui-se que, diante do quantitativo obtido com a pesquisa, faz-se necessária a readequação do número de colaboradores da enfermagem baseada na complexidade da clientela, bem como uma dinâmica mais eficaz para 0 funcionamento desta unidade emergencial.

\section{REFERÊNCIAS}

1. Kurcgant P, Cunha K, Gaidzinski RR. Subsídios para a estimativa de pessoal em enfermagem. Enfoque 1989;17(3):79-81

2. Gaidzinski RR. Dimensionamento de pesso0al em enfermagem. In: K urcgant $P$, organizadora. A dministração em enfermagem. São Paulo: EPU; 1991. p.91-6.

3. A tálaA. Cálculo de pessoal para os serviços paramédicos de um hospital geral de trezentos leitos. Rev Paul Hosp 1978; 26(6).

4. Jung G, OliveiraA, Corral S. D ocumentar a implantação do Therapeutic Intervention Scoring System - TISS no Serviço de Emergência do Hospital M ãe de Deus. Enfermagem A tual 2003; 3(13):27-31.

5. Campedelli MC. Takito C, Sancinetti TR, Benko MA. Cálculo de pessoal em enfermagem: competência da enfermeira. Rev Esc Enfermagem USP 1987; 1(21):3-13.

6. Fugulin FMT, Silva SH, Shimizu HE, Campos FPF. Implantação do sistema de classificação de pacientes na unidade de clínica médica do Hospital Universitário da Universidade de São Paulo. Rev M ed HUUSP 1994; 4(1/2): 63-80.

7. Tomazi NGS, Kalinowski CE, Takii RT, Cisz CM, K ruger V M O, Zen $\mathrm{LM}$. Dimensionamento de recursos humanos em enfermagem. Cogitare Enferm 2001; 6(1):83-9.

8. Camona LM P, Évora Y DM. Sistema de classificação de pacientes: aplicação de um instrumento validado. Rev Esc Enfermagem USP 2002; $1(36): 42-9$.

9. Prevelatto RP. Sistema de classificação de pacientes: instrumento para a prática gerencial do enfermeiro. [monografia]. São Paulo (SP): Universidade de São Paulo; 2000.

10. Figueiredo NM, Machado WCA. Ecosofia e autopoiese no cuidado com o corpo. In: Santos I. et al. Enfermagem fundamental: realidade, questões, soluções. São Paulo: A theneu; 2001. p. 191-210.

11. Fugulin FM, Gaidzinski RR. Horas de assistência de enfermagem: análise comparativa de parâmetros. Nursing 2000; 239(2):30-4.

12. Ribeiro CM . Sistema de classificação de pacientes para provimento de pessoal de enfermagem. [tese]. São Paulo (SP): Universidade de São Paulo; 1972

13. Alcalá MU et al. Cálculo de pessoal: estudo preliminar para estabelecimento de quadro de pessoal de enfermagem na superintendência médico-hospitalar de urgência. São Paulo: Superintendência M édico-Hospitalar de U rgência; 1982.

14. Alves SM et al. Enfermagem: contribuição para um cál culo de recursos humanos naárea. Rio de J aneiro: Coordenadoria de Comunicação Social do INAM PS; 1988.

15. M artins EAP, Haddad M CL. Validação de um instrumento que classifica os pacientes em quatro graus de dependência do cuidado de enfermagem. Rev Latino-am Enferm 2000; 8(2):74-82. 